\title{
Successful Computationally Directed Templating of Metastable Pharmaceutical Polymorphs
}

\section{Published as part of a Crystal Growth and Design virtual special issue Honoring Prof. William Jones and His Contributions to Organic Solid-State Chemistry}

David H. Case, ${ }^{\dagger}$ Vijay K. Srirambhatla, ${ }^{\ddagger}$ Rui Guo, ${ }^{\dagger}$ Rona E. Watson, ${ }^{\dagger}$ Louise S. Price ${ }^{\dagger}$

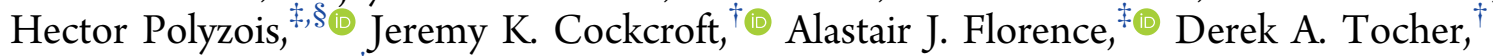
and Sarah L. Price*tio

${ }^{\dagger}$ Department of Chemistry, University College London, 20 Gordon Street, London WC1H 0AJ, U.K.

${ }^{\ddagger}$ EPSRC Future Continuous Manufacturing and Advanced Crystallization Hub, and ${ }^{\S}$ National Physical Laboratory Scottish Hub, University of Strathclyde, Technology and Innovation Centre, 99 George Street, Glasgow, G1 1RD, U.K.

\section{Supporting Information}

ABSTRACT: A strategy of using crystal structure prediction (CSP) methods to determine which, if any, isostructural template could facilitate the first crystallization of a predicted polymorph by vapor deposition is extended to the fenamate family. Mefenamic acid (MFA) and tolfenamic acid (TFA) are used as molecules with minimal chemical differences, whereas flufenamic acid (FFA) shows greater differences in the substituents. The three crystal energy landscapes were calculated, and periodic electronic structure calculations were used to confirm the thermodynamic plausibility of possible isostructural polymorphs to experimentally obtainable crystals of the other molecules. As predicted, a new polymorph, TFA form VI, was found by sublimation onto isomorphous MFA form I, using a recently developed technique. MFA and TFA form a continuous solid solution with the structure of MFA I and TFA VI at the limits, but the isomorphous MFA/FFA solid solution does not extended to a new polymorph of FFA. The novel solid solution structure of TFA and FFA was found, and a new isomorphous polymorph TFA VII was found by sublimation onto this new solid solution template. Sublimation of TFA onto a metal surface at the early stage of deposition gave TFA form VIII. We rationalize the formation of new polymorphs of only TFA.

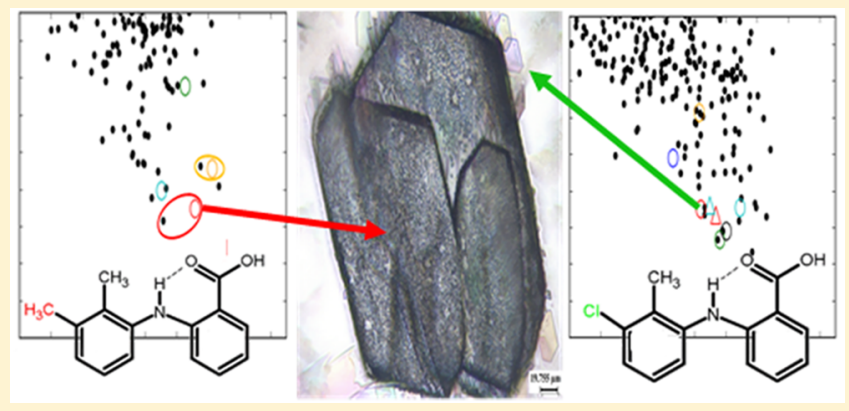

\section{INTRODUCTION}

A knowledge of the solid form landscape, covering at least all polymorphs, solvates, and hydrates, is essential for the design of robust crystallization processes and to avoid the late appearance of unknown competing forms. ${ }^{1-3}$ Extensive efforts in polymorph screening ${ }^{4,5}$ show that the vast majority of molecules are polymorphic, ${ }^{6}$ and yet covering all possible crystallization conditions is not practicable because of the marked effect that impurities or deliberate seeding with related crystals can have on the production of novel polymorphs. ${ }^{7-10}$ Computational crystal structure prediction (CSP) is emerging as a complementary tool to polymorph screening, to help establish whether all polymorphs of interest have been found, ${ }^{11,12}$ but this frequently suggests that more polymorphs are thermodynamically feasible than are experimentally known. ${ }^{13}$ This raises the question as to whether a targeted crystallization experiment can be devised to specifically crystallize the unobserved low energy structures on the computational crystal energy landscape for the first time. ${ }^{13}$
On the basis of the knowledge of relative energies, we have used the possibility of isostructurality between closely related molecules as a route to finding a template to produce the first sample of a CSP predicted polymorph of another molecule. The first successful application of this strategy was in the carbamazepine family, with the production of the first catemeric polymorph of carbamazepine (CBZ V) by sublimation of CBZ onto single crystals of isomorphous dihydrocarbamazepine (DHC II). ${ }^{14}$ (CBZ V has recently been crystallized in another laboratory by a related templating experiment). ${ }^{15}$ While advances in computational methods can provide guidance to select a substrate, experiments to explore all possible orientations between the substrate and overlaying lattice can be very time-consuming. To address this concern, a new approach was developed using the polycrystalline material

Received: May 18, 2018

Revised: July 25, 2018

Published: August 2, 2018 
as substrate. The polycrystalline substrate approach provides access to all possible orientations of the substrate in a singleshot experiment. This strategy was validated by producing the first crystals of a novel polymorph of cyheptamide (CHY III), also using DHC II crystals as the template. ${ }^{16}$

The use of isostructural crystal structures implies that the molecules can adopt such a similar packing that they may well form a solid-solution under experimental conditions. Indeed the discovery of CBZ V was preceded by the demonstration that $\mathrm{CBZ}$ could form catemeric hydrogen bonds in a 50:50 solid solution ${ }^{17}$ of $\mathrm{CBZ} / \mathrm{DHC}$, which is isomorphous with DHC II. In contrast a recent study substituting methyl for $\mathrm{Cl}$ or $\mathrm{Br}$ found solid solutions that were not isomorphous with the pure components. ${ }^{18}$ The differences between surface-templated growth from the vapor, formation of solid solutions, and the use of heterogeneous isostructural crystals for seeding solutions or melts to grow a targeted new form ${ }^{7}$ are important for understanding how similarities between predicted and known crystal structures can be exploited in polymorph discovery.

In this study, we sought to develop the strategy of using CSP to determine possible templates for finding new polymorphs in another family of molecules. The fenamates were chosen as a contrast to the carbamazepine family, in that the fenamate polymorphs differ in conformation and packing of aromatic rings but have the same hydrogen bonding functionality. Since the fenamates are a family of nonsteroidal anti-inflammatories, their polymorphism, transformations, and the effect of different polymorphs on bioavailability have been extensively studied. ${ }^{19,20}$ Many fenamates are highly polymorphic: flufenamic acid (FFA) held the record for nine characterized crystal structures, with forms IV-IX being discovered by polymer templating. ${ }^{21}$ Tolfenamic acid (TFA) has five polymorphs, with III-V being generated by polymer induced heterogeneous nucleation. ${ }^{22,23}$ Mefenamic acid (MFA) has three reported forms, with the metastable form II produced on heating ${ }^{24}$ or by recrystallization under pressure ${ }^{25}$ and with form III being found serendipitously in a cocrystallization experiment. $^{26}$ These polymorphic systems have been extensively used to explore methods of polymorph discovery ${ }^{27}$ and control, including stabilizing metastable forms. ${ }^{28}$ Work seeking to rationalize the observed polymorphism ${ }^{29-31}$ has suggested that the "polymorphophore"22,32 nature arises from the packing of the substituents, ${ }^{33}$ with fenamic acid ${ }^{34}$ being monomorphic. In this study we chose to concentrate on MFA and TFA, as the most similar molecules, as the exchange of $\mathrm{CH}_{3}$ for $\mathrm{Cl}$ involves little difference in atomic volume and is a widely used tool in crystal engineering. ${ }^{35} \mathrm{We}$ also studied FFA to assess the effect of a more significant change in the substituents, which has little effect on the solution properties in polar solvents. $^{36}$

Hence, this paper uses computer prediction and experimental exploration of three of the polymorphic fenamates to study the capabilities of isostructural templating as a route to polymorph discovery by considering the following questions:

(1) Do similar molecules have isomorphous or isostructural crystal structures that are thermodynamically plausible, as generated by CSP methods?

(2) Can the ambient stable polymorphs (MFA I, TFA I, FFA III) be used as templates to generate the first crystals of an isostructural polymorph that has been predicted in (1), by sublimation?
(3) In cases where (2) generates a new polymorph: how structurally similar do the template and novel polymorph have to be? How is this related to the ability to form a solid solution that is isomorphous with both end points? How does the relative stability and kinetic feasibility of the polymorphs of a molecule limit the ability to produce further polymorphs? Can the novel polymorphs be produced by other routes?

\section{EXPERIMENTAL DETAILS}

2.1. Preparation of Solid Solutions. Samples were prepared by mixing pure preformed solutions of the two components in a common solvent (ethanol, diethyl ether, and acetonitrile) and allowing the sample to crystallize by slow evaporation at room temperature. Bulk materials were analyzed by one or both of the techniques of PXRD and ${ }^{1} \mathrm{H}$ NMR spectroscopy to assess the composition of the solid solution material with respect to the input molar ratios. Carefully selected single crystals were analyzed by SCXRD, and the appropriate occupancies were refined to establish composition (Section S7.2). Where solid solutions were prepared from melts, this was done by finely grinding the two components into a single homogeneous powder, which was placed between two microscope slides. The samples were then heated to $175{ }^{\circ} \mathrm{C}$ on a Kofler bench followed by slow cooling to room temperature at $5{ }^{\circ} \mathrm{C} / \mathrm{min}$.

2.2. Procedures for Sublimation Experiments. An array of template crystals were mounted on a glass slide which was then exposed to a vapor of the material to be deposited ${ }^{16}$ produced by placing ca. $50 \mathrm{mg}$ on a Petri dish which was heated to $120^{\circ} \mathrm{C}$ for $24 \mathrm{~h}$. The deposited material was examined by optical microscopy, and individual single crystals were carefully removed and characterized by SCXRD.

2.3. Computational Methodology. The crystal energy landscapes were calculated by generating one million $Z^{\prime}=1$ structures using CrystalPredictor, ${ }^{37}$ in the 59 most common space groups, including the main conformational variable of the fenamates $(\phi$, Figure 1) as a search variable. These structures were further refined<smiles>Cc1cccc(N([IH])c2ccccc2C(=O)O)c1C</smiles>

Figure 1. Fenamates: fenamic (FA), mefenamic (MFA), tolfenamic (TFA), and flufenamic (FFA) acids. The widely varying torsion angle is labeled on FA as $\phi$.

using CrystalOptimizer ${ }^{38}$ to optimize the lattice energies as the sum of the conformational energy penalty, calculated at the PBE0/6$31+G(d)$ level of theory using Gaussian $09,{ }^{39}$ and the intermolecular lattice energy calculated within DMACRYS ${ }^{40}$ using the distributed multipoles calculated by GDMA ${ }^{41}$ and the empirical exp- 6 repulsiondispersion model with the FIT parametrization. ${ }^{42}$ The sensitivity of the low energy crystal structures to the computational model was further investigated by using a polarizable continuum model (PCM, $\varepsilon$ $=3$ ) to calculate the distributed multipoles and intramolecular energy penalty, ${ }^{43}$ and also a rigid-body free energy model. ${ }^{44}$ For selected 


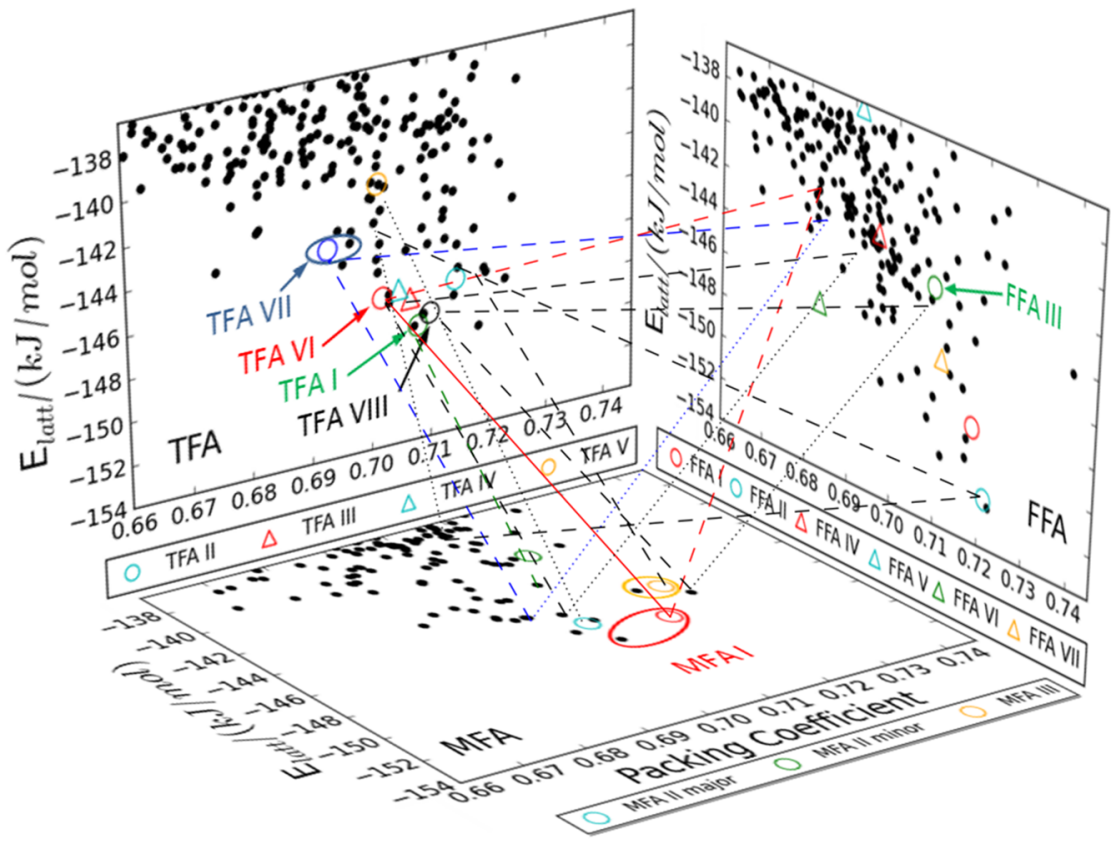

Figure 2. Crystal energy landscapes (CELs) of TFA, MFA, and FFA, where each black dot represents a stable structure in the lattice energy, $E_{\text {latt }}$ evaluated within a polarizable continuum model, generated in the CSP study. Open shapes on each of the three CELs are polymorphs known by the end of the study, shown by circles if $Z^{\prime}=1$, triangles if $Z^{\prime}>1$. CSP generated structures are linked to the observed structure by a larger ellipse if not falling within the symbol. Tie-lines are drawn to link polymorphs to isostructural matches in another CEL: solid lines span structures which are both known polymorphs, dashed lines connect a known polymorph to a hypothetical structure, and dotted lines join structures which are not known for either molecule (although are known polymorphs for the third). Colored lines include a room temperature stable form: red - MFA I structural type, blue - TFA/FFA solid solution (TFA VII), and green - TFA I. The structural types used as templates and the new polymorphs are explicitly labeled, with other known polymorphs included in legends beneath each 2D plot. Full details of the structural matches are in Table S9.

structures, lattice energies were also modeled using CASTEP $^{45}$ with a $\mathrm{PBE}$ functional ${ }^{46}$ and both the $\mathrm{TS}^{47}$ and $\mathrm{MBD}^{* 48}$ dispersion models. The nanocrystal surface calculations were performed using ORIENT. $^{49}$ Further details and analysis are in the Supporting Information.

\section{RESULTS}

3.1. Are There More Fenamate Polymorphs To Be Targeted? The CSP generated crystal energy landscapes show that there are many lattice energy minima that do not correspond to known forms in this extensive search for $Z^{\prime}=1$ structures. Isostructural crystals were identified by having the 15 molecule coordination sphere match (Table S9). This shows that there are far more matches between low energy structures of TFA and MFA than with FFA, and provides an initial estimate of how metastable the structure would be. However, the experiments to use an isostructural template to produce the first nucleation of a new form are limited in that the templating crystal must not transform to a more stable form during the course of the experiment. We have focused therefore on using MFA I, TFA I, and FFA III and solid solution crystals as templates for sublimation. Looking for matches with template structures (Figure 2 and Table S9) shows that there is a structure of TFA that is isomorphous to the most stable form of MFA, and thermodynamically competitive with the metastable polymorphs of TFA. This suggests that TFA should be sublimed onto MFA form I crystals as a potential route to targeted crystallization of a new polymorph of TFA, form VI.

3.2. Targeted Production of the First Crystals of TFA form VI. Subliming TFA onto crystalline MFA form I, using the new methodology, does indeed produce crystals of a new form of TFA, form VI $\left(P \overline{1}, Z^{\prime}=1\right)$, Figures $3, S 13$ and Table S17. The structure has a similar powder X-ray diffraction
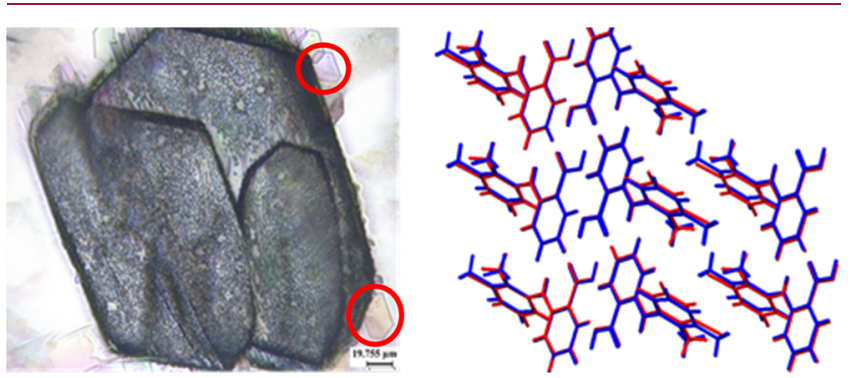

Figure 3. (Left) Plates of TFA VI (circled in red) formed by sublimation of TFA onto MFA I and (right) the overlay of the major component of TFA VI (red) with templating structure MFA I (blue; XYANAC) $\mathrm{RMSD}_{15}=0.214 \AA$.

(PXRD) pattern to TFA IV $\left(P \overline{1}, Z^{\prime}=3\right)$, produced by polymer induced heteronucleation, ${ }^{21}$ suggesting that the templating isomorphous crystal surface directs the TFA molecules into the simpler, slightly more stable lattice of form VI.

The production of TFA VI by sublimation onto MFA I is robust, as it was also produced in a separate laboratory by a modified purification sublimation apparatus. TFA VI can also be obtained by seeding ethanolic solutions of TFA with MFA I crystals and with crystals of FFA I but not FFA III (see Section S7.1.2).

3.3. Solid Solution Extensions of Crystal Structures of These Fenamates. All three binary solid solutions could be formed with a substantial proportion of both molecules. The MFA/TFA and MFA/FFA solid solutions (Sections S7.1.3 and 
S7.1.6) were isomorphous with MFA I. The solid solution of TFA/FFA (Section S7.1.8) is closely related, having very similar layers of hydrogen bonded dimers, but these are related by a screw rather than a translation, giving a $P 2_{1} / c$ structure in contrast to the $P \overline{1}$ structures of MFA/FFA and MFA/TFA. However, the range of composition and methods of formation of these solid solutions differ.

The solid solution of MFA and TFA results from very good molecular matching: not only are both end points now known (MFA I and TFA VI), but careful examination of the molecular ratios in the solid solution, by both single crystal diffraction and $\mathrm{NMR}$ of a $\mathrm{CDCl}_{3}$ solution prepared from the recovered crystals, show approximately the same molar ratio as the starting ethanol solution concentration (Section S7.1.4).

Both FFA solid solutions proved more challenging to produce due to the increased dissimilarity between molecular components. The MFA/FFA solid solution was prepared by crystallization from the melt after failed attempts to obtain the solid solution by crystallization from ethanol solution (Section 7.1.5-6). It is isomorphous with MFA I, but contains only a maximum of $\sim 70 \%$ FFA. The TFA/FFA solid solution can be formed from ethanol solution up to 50\% FFA molar ratio (Section S7.1.8).

3.4. Targeted Production of the First Crystals of TFA VII. The TFA/FFA solid solution provided another new fenamate crystal structure type, which can be readily prepared and is sufficiently stable to be used as template. Adding this to the computational matrix (Table S9) suggested that the TFA end point of the solid solution might be formed by subliming TFA onto the solid solution TFA/FFA. This sublimation experiment generated TFA VII which is isomorphous to the solid solution of TFA/FFA, concomitantly with TFA II crystals (Figure 4).
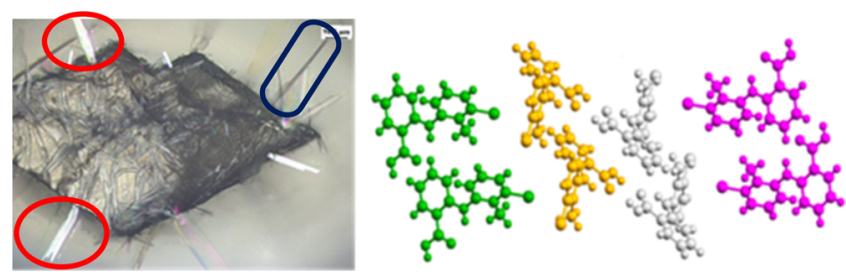

Figure 4. (Left) The new polymorph TFA VII (circled red) formed concomitantly with TFA II (blue), by sublimation onto the isomorphous $P 2_{1} / c$ TFA/FFA solid solution template crystals. (Right) The hydrogen bonded layer, denoted in silver and gold, is identical with that of the $P \overline{1}$ structure TFA VI (which is isomorphous with MFA I, MFA/TFA, and MFA/FFA) with an RMSD $_{12}=0.19 \AA$ but differs in that the layers are not stacked but related by the glide/ screw (purple and green) giving a $\mathrm{T}$ interaction of type $6 \mathrm{a} \beta$ (as defined in Figure S2).

3.5. Control Experiments and Serendipitous Observation of Surface Polymorph TFA VIII. A variety of additional control experiments, subliming MFA, TFA, and FFA onto the template crystals MFA I, TFA I, FFA III, and the TFA/FFA solid solution were attempted. These all resulted in known polymorphs, frequently as mixtures (Section S7.2.1); for example, FFA III and FFA I concomitantly formed on the TFA/FFA solid solution template.

Sublimation of TFA onto a variety of metal and glass surfaces generally gave TFA I, though controlled sublimation ${ }^{50}$ onto copper gives a new form TFA VIII. The structure was determined by PXRD. The powder samples were mixtures of
TFA I $\left(P 2_{1} / c, Z^{\prime}=1\right)$ and a new form TFA VIII $\left(P \overline{1}, Z^{\prime}=1\right)$ which has the same layers stacked in a different way (Figure 5).

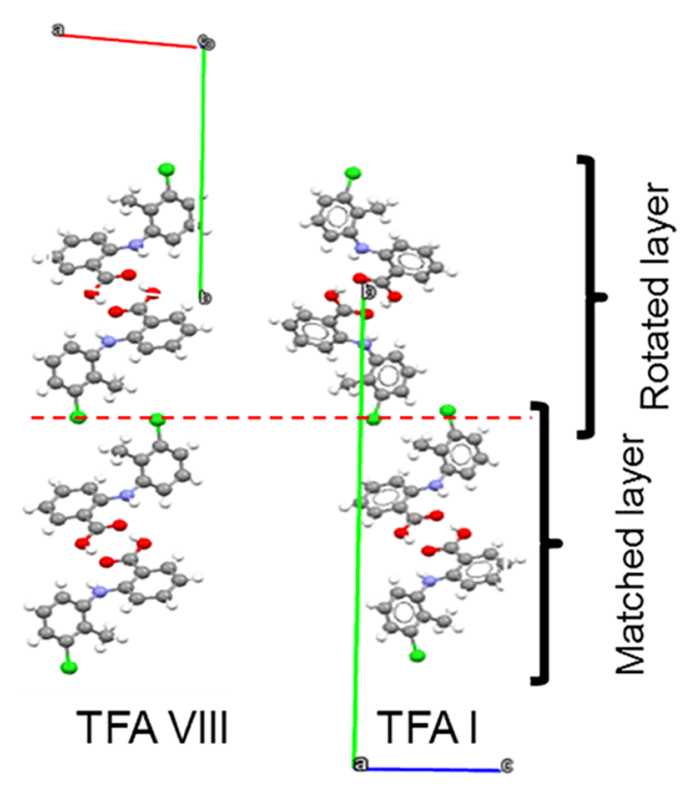

Figure 5. New polymorph TFA VIII has the same layers of dimers as the most stable form at room temperature, TFA I, but stacked by lattice translation rather than a $P 2_{1} / c$ screw axis. The interface between these layers is marked by a dashed red line, and the $b$ axes of the two structures (green), which differ in length by roughly a factor of 2 , are aligned.

The proportion of TFA VIII was greater when less material was sublimed at lower temperatures. Some evidence of polymorph TFA VIII has also been found in samples obtained by sublimation ${ }^{51}$ onto smooth $\mathrm{Ag}$ or $\mathrm{Cu}$ surfaces (Section S8).

\section{DISCUSSION}

4.1. Do Similar Molecules Have Isostructural Structures That Are Thermodynamically Plausible, As Generated by CSP Methods? At the start of this study, the many known polymorphs of the heavily screened fenamates, MFA, TFA, and FFA all had distinct structures, despite MFA and TFA molecules being related by a $\mathrm{Cl} / \mathrm{CH}_{3}$ interchange, which often results in isomorphous crystals, and FFA having one smaller and one similar substituent. The steric similarity accounts for the crystal energy landscapes (Figure 2, Table S9) having matches between most of the observed $Z^{\prime}=$ 1 polymorphs and computer generated structures for the other molecules, within the large $15 \mathrm{~kJ} / \mathrm{mol}$ window. There are many additional computer-generated structures, particularly for FFA. In comparing the relative energies of the matched structures (Table S9), it becomes clear that MFA and TFA have common structures that are similar in relative stability, but FFA structures have a very different energy ranking; i.e., when only the low energy structures, within the range of observed polymorphs, are examined, there are some matches between MFA and TFA, but most matches with FFA are high in energy and vice versa. (An exception is that substituting TFA in FFA II is only slightly less stable than any known TFA polymorph.) On adding the experimental constraint that the templating crystal needs to be stable during the experiment, the most promising unobserved thermodynamically plausible polymorph was experimentally realized as TFA VI. The addition of the TFA/FFA solid solution structure to the matrix suggested that 
the structure which was found as TFA VII was intermediate in stability between the ordered and disordered metastable polymorphs of TFA, and that if MFA could adopt the structure it would be only slightly less stable than any of its known polymorphs. By the completion of this study, we had generated a polymorph of TFA isomorphous with MFA I, namely, TFA VI, and two new fenamate structure types, TFA VIII and the TFA/FFA solid solution with its isomorphous single component end point, TFA VII.

The key to successful templating is the thermodynamic plausibility of a new polymorph, which depends on the possible thermodynamic metastability of experimental polymorphs, and the likely errors in the relative stability as estimated by lattice energy calculations used in the CSP studies. We assessed the computational error by calculating the relative energies of all the experimental structures and the corresponding structures generated by substituting the alternative molecules into these lattices, by a range of dispersion-corrected periodic density functional theory methods, and also by the approximate inclusion of free energy or the effect of polarization as variations on the method used for the CSP study. The results (Section S4) vary as would be expected $^{52,53}$ from the known limitations of all current computational methods for relative polymorph stability and are in reasonable agreement with the limited experimentally established Gibbs free energy differences between polymorphs and evidence for low temperature stability order ${ }^{21,22}$ (Section S4.1). Most calculations, including the most expensive periodic DFT-D calculations with the most theoretically justified dispersion correction (PBE-MBD*) (Figure 6) show that the observed polymorphs are more stable than the unobserved isostructural equivalents and confirm that the templating experiments were targeting metastable structures. We have found new polymorphs of TFA, which are comparable in

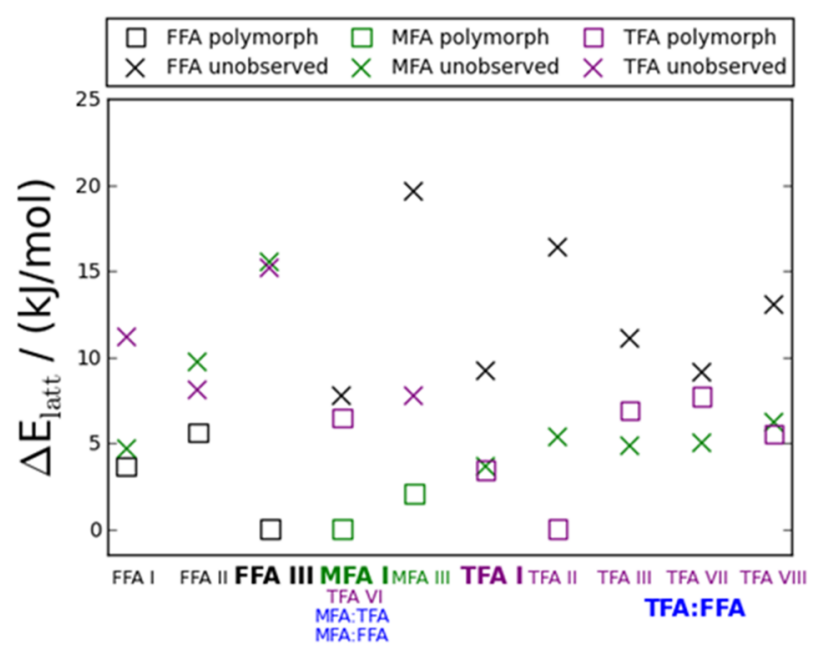

Figure 6. Relative lattice energies of the observed $(\square) Z^{\prime}=1$ nondisordered polymorphs and computational substitutions of the other molecules in the same structures $(X)$; the geometry is optimized at the PBE-TS level, and single point energies are evaluated by PBEMBD*. The energies are relative to the experimentally most stable low temperature polymorphs, i.e., FFA III, MFA I, and TFA II. The color code for the molecules in the crystal structures is followed on the axes, with the solid solutions in blue and the structures used as templates in sublimation experiments in large bold type. See Section S4 for a detailed analysis of relative energies by a variety of computational models and comparison with experimental stabilities. stability with those found by polymer induced heteronucleation, but not the MFA equivalents of TFA polymorphs which would be less stable than the known MFA polymorphs. The FFA equivalents would be highly metastable.

The closeness of the polymorphs' energies reflects the compromise between conformational distortions, the van der Waals dispersion, and $\pi \cdots \pi$ stacking interactions between the aromatic groups, and minor distortions of the common hydrogen bonding motif, with TFA and FFA adding halogen interactions into the mix of contributions which are being balanced. Analyzing the energy differences (Figure 6) helps explain why the most stable polymorphs differ and why there are not more isostructural structures adopted by these chemically similar molecules. The packing of FFA III is markedly more stable for FFA than it would be for TFA or MFA, as it adopts a conformation in which there is a steric clash between the additional methyl groups of MFA and TFA (Figure 7). All three molecules have a double well in the

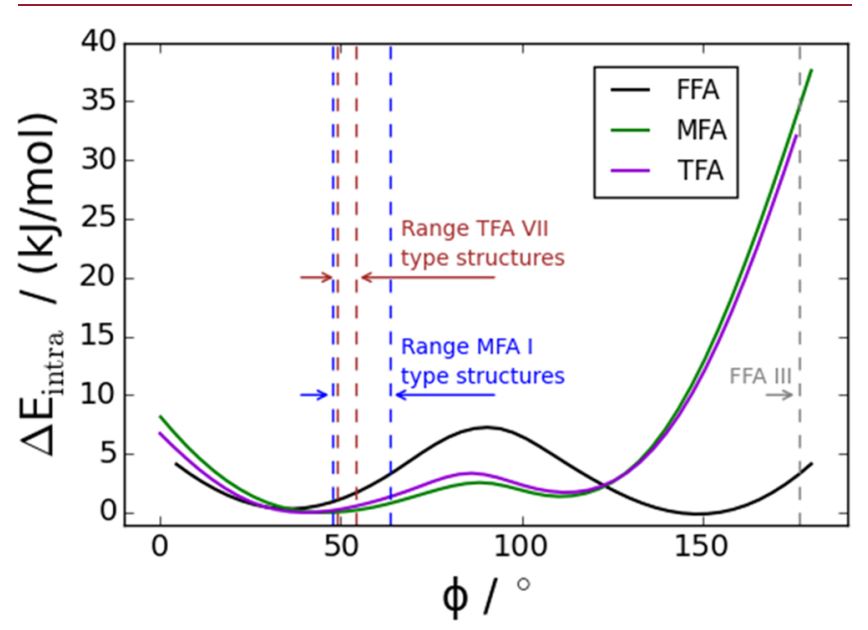

Figure 7. Intramolecular (conformational) energy $\left(\Delta E_{\text {intra }}\right)$ at the PBE0/6-31+G(d) level of theory for the three molecules as a function of $\phi$. The range of dihedral angles observed in solid solutions and polymorphs which are isostructural with MFA I and TFA VII are shown, as is the dihedral angle found in FFA III. All experimental angles are listed in Table $S 1$ and shown in the individual conformational profiles, Figure S3.

conformational energy, which can be attributed to the compromise between conjugation of the two aromatic rings favoring planar structures and the steric clash of the substituents $^{54}$ and gives a similar small barrier when $\phi=0^{\circ}$ corresponding to only proximity of hydrogen atoms. However, for FFA, the barrier for perpendicular rings is high and conformations with $\phi \approx 180^{\circ}$ accessible, whereas TFA and MFA have a lower energy barrier for perpendicular rings and a very high barrier around a planar conformation due to close approach of the methyl substituents. Thus, MFA and TFA cannot adopt the structures of FFA III, IV, VI, and VII because these contain at least one molecule in a high energy conformation (Figure S3). TFA and MFA have crystalline conformations in both wells, and there is only a small energy barrier between all observed conformations in isolation. The differences in low energy conformations may explain why MFA can be incorporated as an impurity into FFA I ${ }^{55,56}$ more readily than FFA III and that the MFA impurity inhibits the polymorphic transition from FFA I to III. In the case of FFA growth on other templates, the large barrier for FFA III 

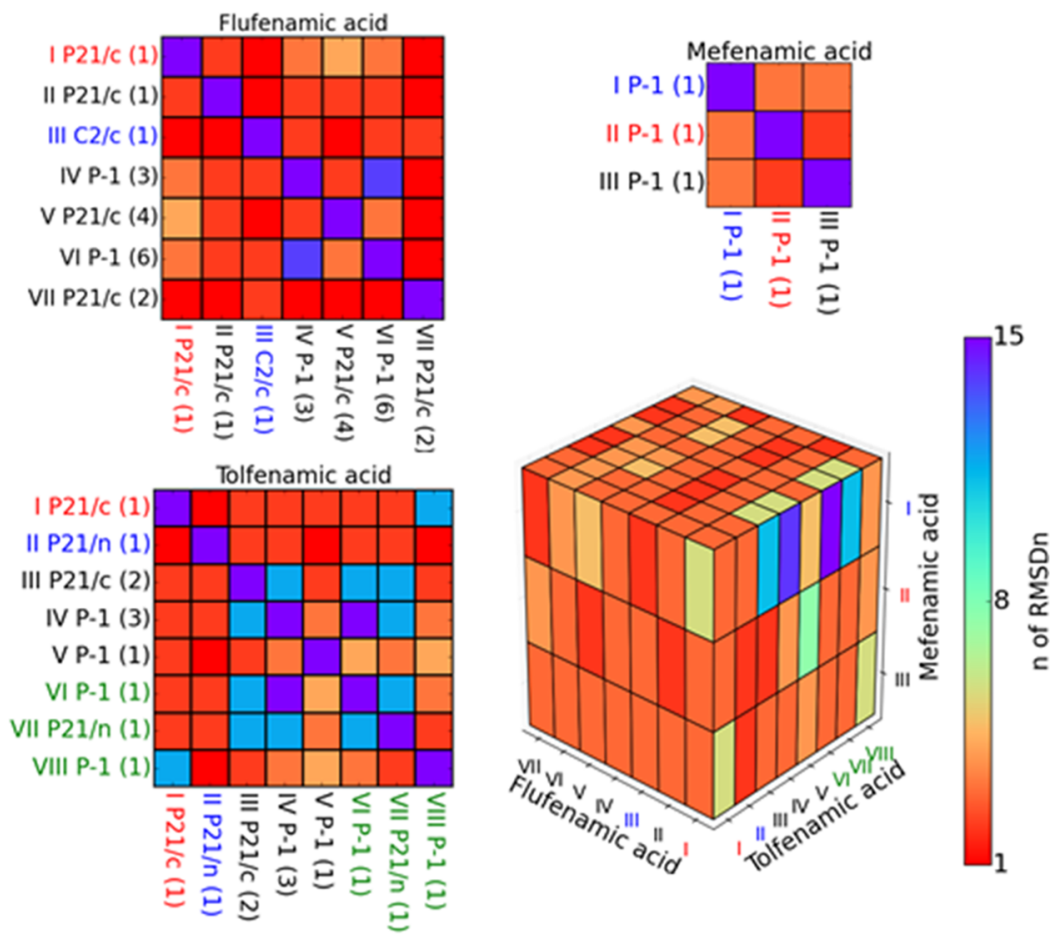

Figure 8. Similarities between the polymorphs of the fenamates shown as $n$, the number of molecules in the best cluster overlay between the two structures $(n \leq 15)$. Forms labeled in blue are the most stable at low temperatures, those in red at high temperature, and novel structures reported in this work in green. The comparisons of all the polymorphs of a molecule with others of the same molecule are shown as matrices, labeled with space group $\left(Z^{\prime}\right)$. The comparisons of polymorphs of one molecule with those of another are all shown on the cubic graph. Numerical values of RMSDn are included in Tables S2-S7. FFA VIII with $Z^{\prime}=8.5$ is not included.

molecules to change conformation to the shape adopted by MFA and TFA polymorphs may also provide a kinetic barrier to forming new FFA polymorphs.

The other component of the lattice energies is the intermolecular energy. The carboxylic acid $R_{2}^{2}(8)$ hydrogen bond is the same in all structures, and so the main causes of the energy differences come from the packing of the substituted aromatic rings. The classification in Figure S2 of these interactions shows that the MFA I structure has methyl to methyl interactions, which can have one $\mathrm{CH}_{3}$ replaced by $\mathrm{Cl}$ for TFA, or plausibly be adopted by FFA. This accounts for MFA I being the most commonly observed structural type, with the MFA/TFA solid solution existing continuously between the MFA I and TFA VI end points, and the MFA/ FFA solid solution accommodating up to $70 \%$ FFA in this structure. All MFA polymorphs have the pair of methyl groups adjacent. MFA and TFA are similar in that the nonacidic rings are often approximately coplanar, but TFA has a wider range of contacts, with those where the $\mathrm{Cl}$ is near an aromatic hydrogen being the more stable. FFA appears to have a preference for structures in which $\mathrm{CF}_{3}$ groups are aligned. There are some crystal structures of TFA and FFA where the substituents are pointing into the aromatic ring, including TFA VIII. This is the main packing difference between the MFA solid solution structures (MFA I) and the TFA/FFA solid solution (Figure 4). Overall, although the molecular differences can be used to rationalize the differences in the low energy crystal structures, the different contributions have to be balanced using high-level calculations.

4.2. Use of Templates To Generate the First Crystals of a Predicted Isostructural Polymorph. The production of TFA VI by sublimation onto the isomorphous template of
MFA I is a clear success of the principle of using CSP to establish that there is an unobserved low energy polymorph that is isostructural with an available template that can be used in the sublimation experiment. The two molecules also form a solid solution with the same structure, with the molecules in the input ratio. This success in getting TFA to adopt the most stable structure for MFA seemed likely from the $\mathrm{Cl} / \mathrm{CH}_{3}$ exchange principle and is fully consistent with the calculated lattice energies. The robustness of the novel TFA VI is shown by its being formed in distinct sublimation experiments and by seeding ethanolic solutions.

The second new polymorph produced by sublimation onto a template is the formation of TFA VII on the TFA/FFA solid solution. This structure differs from the MFA I type, purely in the packing of the layers, reflecting the differences in the substituents. Thus, TFA can adopt either layer packing, but neither MFA nor FFA can, which is consistent with TFA being in both solid solution structures, but FFA being only partially absorbed into both solid solutions, and MFA I being the only solid solution structure for MFA.

4.3. Thermodynamic and Kinetic Feasibility of the Polymorphs. All the new polymorphs of TFA are metastable, and hence their formation is in kinetic competition with the more stable structures, and the time taken for characterization requires a slow transformation rate (TFA VI crystals were stable for more than a week and TFA VII for more than a day). There is no clear dividing line in the allowed degree of thermodynamic metastability (Figure 6); for example, a highly metastable form of MFA could have been templated by TFA I or the TFA/FFA solid solution, both of which only gave MFA I. The comparison of the observed polymorphs (Figure 8) shows a high degree of similarity between MFA I, the most 
stable form, and the metastable TFA polymorphs, so MFA is unlikely to be long-lived in an isostructural equivalent to TFA III, IV, or VII. The challenges in obtaining the known metastable forms of MFA as single crystals have been attributed to the ease of conversion to the stable form. ${ }^{25} \mathrm{~A}$ study of the epitaxial growth of MFA onto FFA I and FFA III ${ }^{56}$ found that the substrate affected the rate and orientation of crystalline growth, but it was MFA I that was formed.

4.4. Implications for the Design/Choice of Templates. The successful selection of templating crystals by modeling raises the question: when a CSP study shows that a molecule has unobserved yet potentially important thermodynamically plausible polymorphs, how you find a suitable template? Searching the CSD for an isostructural match to the CSP generated low energy structure, ignoring small molecular differences, might suggest a suitable template crystal for the targeted structure. The ability of the two molecules to form solid solutions also appears to be a useful indicator that the isostructurality is close enough. However, when the solid solution range is not continuous across all relative concentrations, as observed for FFA, sublimation has not produced the isomorphous FFA end-point. Practically, the sensitivity of the relative lattice energies to molecular changes, even as subtle as changing $\mathrm{CH}_{3}$ for $\mathrm{Cl}$, shown by the CSP studies (Figure 2), implies that it is likely to be rare that these conditions of close isostructurality are met. The kinetic effects that are operational in templating the formation of new polymorphs occur at the surface, and so we might hope that a suitable template crystal need only have some surfaces that could act as a template. We have explored the compatibility between different crystal surfaces by docking nanocrystals ${ }^{57}$ (of 25 molecules cut from the optimized target crystal structure) onto the idealized dominant crystal surfaces of the templates. (Section S5). These calculations show (Figure 9) that TFA can interact very strongly with the MFA I template surface supporting the formation of TFA VI by templating. In contrast FFA cannot form a stable structure that is closely isostructural to MFA I

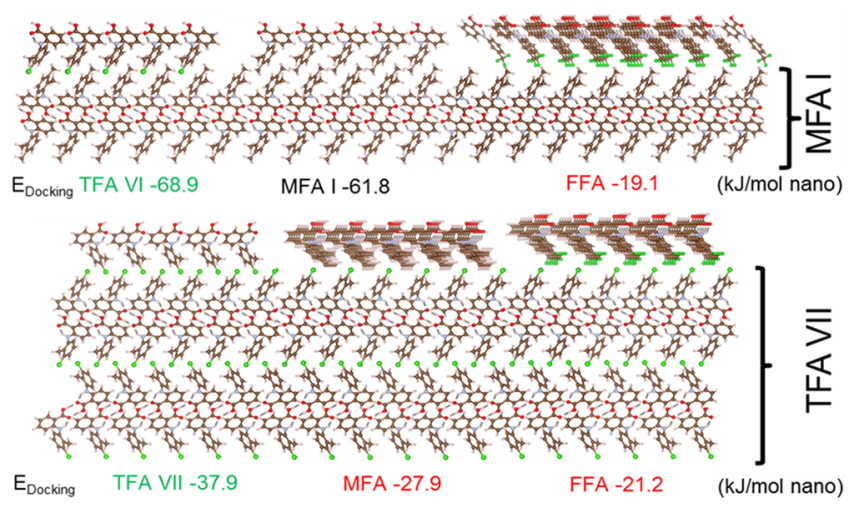

Figure 9. Dominant surfaces of the template crystals. Top: $P \overline{1}$ MFA I and bottom $P 2_{1} / c$ TFA VII representing the TFA/FFA solid solution, showing the docking of 25 molecule nanocrystals of the same structure composed of (left to right) TFA, MFA, and FFA. The energy of docking the nanocrystal is color coded green for successful templating experiments, black for previously known pure polymorphs, and red for cases where templating did not produce a structure corresponding to the nanocrystal. The dominant interactions in the docking are the broadside substituent interactions (1a Figure S2) for the $P \overline{1}$ structure (top) and the T-shaped interaction (6a $\beta$ Figure S2) for the $P 2_{1} / c$ structure (bottom).
$\left(\mathrm{RMSD}_{14}=0.7 \AA\right.$ Table S9), and so the registry between the nanocrystal of FFA in this structure with the MFA I surface is poor. The results for the templating onto the TFA/FFA solid solution are less clear-cut, but the TFA VII structure that is templated by this crystal does have the strongest interaction (Figure 9 Table S12). The nanocrystal results are consistent with the bulk crystal energy predictions for templating and the experimental results, and so this approach may be useful for exploring surface matches that do not involve isostructural crystals. The idea of using nanocrystal to surface interactions has been used to establish the compatibility of two surfaces in various contexts, including determining possible agglomerating faces, ${ }^{57}$ accounting for surface templating favoring a particular hydration product ${ }^{58}$ or cocrystal growth, ${ }^{59}$ and establishing epitaxy $^{60,61}$ as a quantification of two crystals sharing the same surface structure. ${ }^{62}$ A smaller cluster can explain why seeding solutions with MFA I and FFA I produces TFA VI (Section S6). The similar surface is expected to lower the nucleation barrier to forming the new, possibly metastable form, ${ }^{62}$ and the nanocrystal represents establishing the new structure. These simulations are far from realistic, as if the nanocrystal docking onto unrelaxed surfaces represented the only templating mechanism, this would result in only a lateral continuation of the crystal growth. In our experiments, the fastest growing crystals stick out from the crystal faces vertically (Figure 3 (left) and Figure 4 (left)), though lateral crystals were also seen, as in the carbamazepine family templating experiments. ${ }^{16}$

Different organic surfaces or functional groups have long been associated with fenamate polymorphism, with many of the TFA and FFA polymorphs being discovered by polymer induced heteronucleation. ${ }^{21}$ FFA can be used as an additive to produce the metastable MFA II, ${ }^{63}$ and MFA III was found serendipitously in a failed cocrystallization with adenine. ${ }^{26}$ FFA $\mathrm{V}$ can be produced by addition of a salt additive, ${ }^{63}$ and we have also observed FFA $\mathrm{V}$ being formed when attempting to prepare a TFA/FFA solid solution with a high proportion of FFA (Table S16). The geometry of cellulose nanopores affects whether MFA I or II is crystallized. $^{28}$ Self-assembled monolayers have templated mixtures of known forms of TFA and FFA, including TFA "Form X", which seems to be a mixture of TFA I and II. ${ }^{64}$ The observation of TFA VIII at the early stages of deposition onto copper surfaces, which lack the molecular structure of the isostructural templates, indicates other substrates will have an effect. However, our approach leads to the design of templates to target the discovery of a specific isostructural CSP predicted polymorph, and combines both functional group and crystal geometry matching.

The fenamate molecules have an irregular shape, and the CSP crystal energy landscapes show that all three molecules have other thermodynamically feasible structures. Finding or eliminating them from being possible polymorphs is challenging to our understanding of what controls the crystallization of flexible drug molecules. The van der Waals contacts of the flexible fenamates contrast with the use of isostructural templating to produce different hydrogen bonding motifs in the carbamazepine family of rigid molecules. ${ }^{16}$ This shows that the methodology could have applicability across a wide range of pharmaceutical molecules, given that many closely related molecules are likely to have been crystallized in drug discovery. 


\section{CONCLUSION}

By studying the isostructurality of known and computer generated crystal structures of three fenamate molecules, we have produced two new polymorphs of TFA, with a third being found by sublimation onto an inorganic surface. This is for a molecule that has been heavily studied in developing polymorph screening methods. ${ }^{23}$ The methodology originally used in the carbamazepine family, of subliming molecules onto isomorphous templates to produce the first nucleation of a predicted polymorph, has produced the first isomorphous pair of polymorphs (TFA VI and MFA I) within the fenamate family. The generation of solid solutions has produced a new fenamate structural type, the TFA/FFA solid solution, whose single component end point could be obtained by sublimation as TFA VII. There are more similarities in the current set of polymorph structures between MFA and TFA than between either and FFA (Figure 8). The differences in the conformational profile of the molecules, and the variety of favored packings of the substituents of the aromatic ring, account for the difference in the relative stabilities of the different packings for the different molecules. However, the subtle balance between conformational energies and steric requirements to provide dense packing with different substituents is such that controlling the crystallization of these fenamates is an experimental and computational challenge. Since this arises from the compromises between the shallow potential wells for changing conformation and the weak intermolecular interactions, this behavior is likely to be typical of polymorphism in many pharmaceuticals.

This work shows that the targeted generation of new polymorphs by isomorphous templating can be extended to flexible molecules, and weaker intermolecular interactions, but is likely to require molecules with sufficient similarity to form at least a partial isomorphous solid solution. This is a significant step forward in the ability to design specific experiments to produce the first crystals of pharmaceutical polymorphs predicted by CSP.

\section{ASSOCIATED CONTENT}

\section{S Supporting Information}

The Supporting Information is available free of charge on the ACS Publications website at DOI: 10.1021/acs.cgd.8b00765.

Full details of all the computational modeling and experimental work (PDF)

\section{Accession Codes}

CCDC 1842473-1842474 (TFA/FFA_60_40 and TFA/ FFA_53_47), 1842476 (TFA_VII), 1842478 (FFA/ MFA $70-30$ ), 1842699 (TFA VIII), and 1843203-1843206 (TFA_VI, MFA/TFA_0.2_0.8, MFA/TFA_0.5_0.5, MFA/ TFA_0.4_0.6) contain the supplementary crystallographic data for this paper. These data can be obtained free of charge via www.ccdc.cam.ac.uk/data_request/cif, or by emailing data request@ccdc.cam.ac.uk, or by contacting The Cambridge Crystallographic Data Centre, 12 Union Road, Cambridge CB2 1EZ, UK; fax: +44 1223336033.

\section{AUTHOR INFORMATION}

\section{Corresponding Author}

*Telephone: +44(0)207679 4622; e-mail: s.l.price@ucl.ac.uk. ORCID $\odot$

Vijay K. Srirambhatla: 0000-0002-4492-7567
Hector Polyzois: 0000-0002-7630-7063

Jeremy K. Cockcroft: 0000-0002-4954-651X

Alastair J. Florence: 0000-0002-9706-8364

Sarah L. Price: 0000-0002-1230-7427

\section{Funding}

RCUK (EPSRC) EP/K039229/1 and EP/L000202.

\section{Notes}

The authors declare no competing financial interest.

\section{ACKNOWLEDGMENTS}

We thank Profs. Pantelides and Adjiman for the use of CrystalPredictor and CrystalOptimizer codes, Dr. Christoph Salzmann and NPL for the controlled sublimation apparatus onto metal surfaces, and Dr. Martin Vickers for initial structure solution of TFA VII. H.P. acknowledges the NPL Scottish Hub for funding and thanks Dr. Alex Shard, Magdalena Wywijas, and Steve Spencer for their input and support with the sublimation studies performed at NPL and Naresh Kumar for preparing the smooth substrates used in these experiments. This work was supported by EPSRC (EP/K039229/1). ARCHER time was provided via our membership of the UK's HEC Materials Chemistry Consortium, which is funded by EPSRC (EP/L000202).

\section{ABBREVIATIONS}

FA, fenamic acid; MFA, mefenamic acid; TFA, tolfenamic acid; FFA, flufenamic acid; CSP, crystal structure prediction; CEL, crystal energy landscape; PXRD, powder X-ray diffraction; SCXRD, single crystal X-ray diffraction

\section{REFERENCES}

(1) Chen, J.; Sarma, B.; Evans, J. M. B.; Myerson, A. S. Pharmaceutical Crystallization. Cryst. Growth Des. 2011, 11, 887-895.

(2) Sun, C. C. Material Science Tetrahedron-A Useful Tool for Pharmaceutical Research and Development. J. Pharm. Sci. 2009, 98, $1671-1687$

(3) Bucar, D. K.; Lancaster, R. W.; Bernstein, J. Disappearing Polymorphs Revisited. Angew. Chem., Int. Ed. 2015, 54, 6972-6993.

(4) Newman, A. Specialized Solid Form Screening Techniques. Org. Process Res. Dev. 2013, 17, 457-471.

(5) Braun, D. E.; Lingireddy, S. R.; Beidelschies, M. D.; Guo, R.; Muller, P.; Price, S. L.; Reutzel-Edens, S. M. Unraveling Complexity in the Solid Form Screening of a Pharmaceutical Salt: Why so Many Forms? Why so Few? Cryst. Growth Des. 2017, 17, 5349-5365.

(6) Cruz-Cabeza, A. J.; Reutzel-Edens, S. M.; Bernstein, J. Facts and fictions about polymorphism. Chem. Soc. Rev. 2015, 44, 8619-8635.

(7) Bucar, D. K.; Day, G. M.; Halasz, I.; Zhang, G. G. Z.; Sander, J. R. G.; Reid, D. G.; MacGillivray, L. R.; Duer, M. J.; Jones, W. The curious case of (caffeine).(benzoic acid): how heteronuclear seeding allowed the formation of an elusive cocrystal. Chemical Science 2013, 4, 4417-4425.

(8) Lancaster, R. W.; Harris, L. D.; Pearson, D. Fifty-year old samples of progesterone demonstrate the complex role of synthetic impurities in stabilizing a metastable polymorph. CrystEngComm 2011, 13, 1775-1777.

(9) Padrela, L.; Zeglinski, J.; Ryan, K. M. Insight into the Role of Additives in Controlling Polymorphic Outcome: A CO2-Antisolvent Crystallization Process of Carbamazepine. Cryst. Growth Des. 2017, 17, 4544-4553.

(10) Coles, S. J.; Ellis, A. L.; Leung, K.; Sarson, J.; Threlfall, T. L.; Tizzard, G. J. Relationships between the racemic structures of substituted mandelic acids containing 8-and 10-membered hydrogen bonded dimer rings. CrystEngComm 2014, 16, 10816-10823. 
(11) Price, S. L.; Braun, D. E.; Reutzel-Edens, S. M. Can computed crystal energy landscapes help understand pharmaceutical solids? Chem. Commun. 2016, 52, 7065-7077.

(12) Price, S. L.; Reutzel-Edens, S. M. The potential of computed crystal energy landscapes to aid solid-form development. Drug Discovery Today 2016, 21, 912-923.

(13) Price, S. L. Why don't we find more polymorphs? Acta Crystallogr., Sect. B: Struct. Sci., Cryst. Eng. Mater. 2013, 69, 313-328. (14) Arlin, J. B.; Price, L. S.; Price, S. L.; Florence, A. J. A strategy for producing predicted polymorphs: catemeric carbamazepine form V. Chem. Commun. 2011, 47, 7074-7076.

(15) Kamali, N.; O’Malley, C.; Mahon, M. F.; Erxleben, A.; McArdle, P. Use of Sublimation Catalysis and Polycrystalline Powder Templates for Polymorph Control of Gas Phase Crystallization. Cryst. Growth Des. 2018, 18, 3510-3516.

(16) Srirambhatla, V. K.; Guo, R.; Price, S. L.; Florence, A. J. Isomorphous template induced crystallisation: a robust method for the targeted crystallisation of computationally predicted metastable polymorphs. Chem. Commun. 2016, 52, 7384-7386.

(17) Florence, A. J.; Leech, C. K.; Shankland, N.; Shankland, K.; Johnston, A. Control and prediction of packing motifs: a rare occurrence of carbamazepine in a catemeric configuration. CrystEngComm 2006, 8, 746-747.

(18) Chakraborty, S.; Joseph, S.; Desiraju, G. R. Probing the Crystal Structure Landscape by Doping: 4-Bromo, 4-Chloro, and 4Methylcinnamic Acids. Angew. Chem., Int. Ed. 2018, 57, 9279-9283.

(19) Aguiar, A. J.; Zelmer, J. E. Dissolution Behavior Of Polymorphs Of Chloramphenicaol Palmitate And Mefenamic Acid. J. Pharm. Sci. 1969, 58, 983-987.

(20) Surov, A. O.; Terekhova, I. V.; Bauer-Brandl, A.; Perlovich, G. L. Thermodynamic and Structural Aspects of Some Fenamate Molecular Crystals. Cryst. Growth Des. 2009, 9, 3265-3272.

(21) Lopez-Mejias, V.; Kampf, J. W.; Matzger, A. J. Nonamorphism in Flufenamic Acid and a New Record for a Polymorphic Compound with Solved Structures. J. Am. Chem. Soc. 2012, 134, 9872-9875.

(22) Lopez-Mejias, V.; Kampf, J. W.; Matzger, A. J. Polymer-Induced Heteronucleation of Tolfenamic Acid: Structural Investigation of a Pentamorph. J. Am. Chem. Soc. 2009, 131, 4554-4555.

(23) Pfund, L. Y.; Matzger, A. J. Towards Exhaustive and Automated High-Throughput Screening for Crystalline Polymorphs. ACS Comb. Sci. 2014, 16, 309-313.

(24) Panchagnula, R.; Sundaramurthy, P.; Pillai, O.; Agrawal, S.; Raj, Y. A. Solid-state characterization of mefenamic acid. J. Pharm. Sci. 2004, 93, 1019-1029.

(25) Abbas, N.; Oswald, I. D. H.; Pulham, C. R. Accessing Mefenamic Acid Form II through High-Pressure Recrystallisation. Pharmaceutics 2017, 9, 16.

(26) SeethaLekshmi, S.; Guru Row, T. N. Conformational Polymorphism in a Non-steroidal Anti-inflammatory Drug, Mefenamic Acid. Cryst. Growth Des. 2012, 12, 4283-4289.

(27) Alvarez, A. J.; Singh, A.; Myerson, A. S. Polymorph Screening: Comparing a Semi-Automated Approach with a High Throughput Method. Cryst. Growth Des. 2009, 9, 4181-4188.

(28) Lopez-Mejias, V.; Myerson, A. S.; Trout, B. L. Geometric Design of Heterogeneous Nucleation Sites on Biocompatible Surfaces. Cryst. Growth Des. 2013, 13, 3835-3841.

(29) Du, W.; Cruz-Cabeza, A. J.; Woutersen, S.; Davey, R. J.; Yin, Q. Can the study of self-assembly in solution lead to a good model for the nucleation pathway? The case of tolfenamic acid. Chemical Science 2015, 6, 3515-3524.

(30) Mattei, A.; Li, T. Polymorph Formation and Nucleation Mechanism of Tolfenamic Acid in Solution: An Investigation of Prenucleation Solute Association. Pharm. Res. 2012, 29, 460-470.

(31) Mattei, A.; Li, T. L. Interplay between molecular conformation and intermolecular interactions in conformational polymorphism: A molecular perspective from electronic calculations of tolfenamic acid. Int. J. Pharm. 2011, 418, 179-186.
(32) Lutker, K. M.; Tolstyka, Z. P.; Matzger, A. J. Investigation of a privileged polymorphic motif: A dimeric ROY derivative. Cryst. Growth Des. 2008, 8, 136-139.

(33) Uzoh, O. G.; Cruz-Cabeza, A. J.; Price, S. L. Is the Fenamate Group a Polymorphophore? Contrasting the Crystal Energy Landscapes of Fenamic and Tolfenamic Acids. Cryst. Growth Des. 2012, 12, 4230-4239.

(34) Lopez-Mejias, V.; Matzger, A. J. Structure-Polymorphism Study of Fenamates: Toward Developing an Understanding of the Polymorphophore. Cryst. Growth Des. 2015, 15, 3955-3962.

(35) Theocharis, C. R.; Desiraju, G. R.; Jones, W. The Use Of Mixed-Crystals For Engineering Organic Solid-State Reactions Application To Benzylbenzylidenecyclopentanones. J. Am. Chem. Soc. 1984, 106, 3606-3609.

(36) Lee, E. H.; Byrn, S. R.; Pinal, R. The solution properties of mefenamic acid and a closely related analogue are indistinguishable in polar solvents but significantly different in nonpolar environments. J. Pharm. Sci. 2012, 101, 4529-4539.

(37) Karamertzanis, P. G.; Pantelides, C. C. Ab initio crystal structure prediction. II. Flexible molecules. Mol. Phys. 2007, 105, 273-291.

(38) Kazantsev, A. V.; Karamertzanis, P. G.; Adjiman, C. S.; Pantelides, C. C. Efficient Handling of Molecular Flexibility in Lattice Energy Minimization of Organic Crystals. J. Chem. Theory Comput. 2011, 7, 1998-2016.

(39) Frisch, M. J.; Trucks, G. W.; Schlegel, H. B.; Scuseria, G. E.; Robb, M. A.; Cheeseman, J. R.; Scalmani, G.; Barone, V.; Mennucci, B.; Petersson, G. A.; Nakatsuji, H.; Caricato, M.; Li, X.; Hratchian, H. P.; Izmaylov, A. F.; Bloino, J.; Zheng, G.; Sonnenberg, J. L.; Hada, M.; Ehara, M.; Toyota, K.; Fukuda, R.; Hasegawa, J.; Ishida, M.; Nakajima, T.; Honda, Y.; Kitao, O.; Nakai, H.; Vreven, T.; Montgomery, J. A., Jr.; Peralta, J. E.; Ogliaro, F.; Bearpark, M.; Heyd, J. J.; Brothers, E.; Kudin, K. N.; Staroverov, V. N.; Kobayashi, R.; Normand, J.; Raghavachari, K.; Rendell, A.; Burant, J. C.; Iyengar, S. S.; Tomasi, J.; Cossi, M.; Rega, N.; Millam, J. M.; Klene, M.; Knox, J. E.; Cross, J. B.; Bakken, V.; Adamo, C.; Jaramillo, J.; Gomperts, R.; Stratmann, R. E.; Yazyev, O.; Austin, A. J.; Cammi, R.; Pomelli, C.; Ochterski, J. W.; Martin, R. L.; Morokuma, K.; Zakrzewski, V. G.; Voth, G. A.; Salvador, P.; Dannenberg, J. J.; Dapprich, S.; Daniels, A. D.; Farkas, O.; Foresman, J. B.; Ortiz, J. V.; Cioslowski, J.; Fox, D. J. Gaussian 09; Gaussian, Inc.: Wallingford, CT, USA, 2009.

(40) Price, S. L.; Leslie, M.; Welch, G. W. A.; Habgood, M.; Price, L. S.; Karamertzanis, P. G.; Day, G. M. Modelling Organic Crystal Structures using Distributed Multipole and Polarizability-Based Model Intermolecular Potentials. Phys. Chem. Chem. Phys. 2010, 12, $8478-8490$

(41) Stone, A. J. Distributed multipole analysis: Stability for large basis sets. J. Chem. Theory Comput. 2005, 1, 1128-1132.

(42) Coombes, D. S.; Price, S. L.; Willock, D. J.; Leslie, M. Role of Electrostatic Interactions in Determining the Crystal Structures of Polar Organic Molecules. A Distributed Multipole Study. J. Phys. Chem. 1996, 100, 7352-7360.

(43) Cooper, T. G.; Hejczyk, K. E.; Jones, W.; Day, G. M. Molecular Polarization Effects on the Relative Energies of the Real and Putative Crystal Structures of Valine. J. Chem. Theory Comput. 2008, 4, 17951805.

(44) Nyman, J.; Day, G. M. Static and lattice vibrational energy differences between polymorphs. CrystEngComm 2015, 17, 51545165.

(45) Clark, S. J.; Segall, M. D.; Pickard, C. J.; Hasnip, P. J.; Probert, M. J.; Refson, K.; Payne, M. C. First principles methods using CASTEP. Z. Kristallogr. - Cryst. Mater. 2005, 220, 567-570.

(46) Perdew, J. P.; Burke, K.; Ernzerhof, M. Generalized gradient approximation made simple. Phys. Rev. Lett. 1996, 77, 3865-3868.

(47) Tkatchenko, A.; Scheffler, M. Accurate Molecular Van Der Waals Interactions from Ground-State Electron Density and FreeAtom Reference Data. Phys. Rev. Lett. 2009, 102, 073005. 
(48) Tkatchenko, A.; DiStasio, R. A. J.; Car, R.; Scheffler, M. Accurate and efficient method for many-body van der Waals interactions. Phys. Rev. Lett. 2012, 108, 236402-236402.

(49) Stone, A. J.; Dullweber, A.; Engkvist, O.; Fraschini, E.; Hodges, M. P.; Meredith, A. W.; Nutt, D. R.; Popelier, P. L. A.; Wales, D. J. ORIENT: A Program for Studying Interactions between Molecules, 4.8.29; University of Cambridge, 2015.

(50) Shephard, J. J.; Evans, J. S. O.; Salzmann, C. G. Structural Relaxation of Low-Density Amorphous Ice upon Thermal Annealing. J. Phys. Chem. Lett. 2013, 4, 3672-3676.

(51) Shard, A. G.; Havelund, R.; Spencer, S. J.; Gilmore, I. S.; Alexander, M. R.; Angerer, T. B.; Aoyagi, S.; Barnes, J.-P.; Benayad, A.; Bernasik, A.; Ceccone, G.; Counsell, J. D. P.; Deeks, C.; Fletcher, J. S.; Graham, D. J.; Heuser, C.; Lee, T. G.; Marie, C.; Marzec, M. M.; Mishra, G.; Rading, D.; Renault, O.; Scurr, D. J.; Shon, H. K.; Spampinato, V.; Tian, H.; Wang, F.; Winograd, N.; Wu, K.; Wucher, A.; Zhou, Y.; Zhu, Z. Measuring Compositions in Organic Depth Profiling: Results from a VAMAS Interlaboratory Study. J. Phys. Chem. B 2015, 119, 10784-10797.

(52) Beran, G. J. O. Modeling Polymorphic Molecular Crystals with Electronic Structure Theory. Chem. Rev. 2016, 116, 5567-5613.

(53) Zen, A.; Brandenburg, J. G.; Klimes, J.; Tkatchenko, A.; Alfe, D.; Michaelides, A. Fast and accurate quantum Monte Carlo for molecular crystals. Proc. Natl. Acad. Sci. U. S. A. 2018, 115, 17241729.

(54) Uzoh, O. G.; Galek, P. T. A.; Price, S. L. Analysis of the conformational profiles of fenamates shows route towards novel, higher accuracy, force-fields for pharmaceuticals. Phys. Chem. Chem. Phys. 2015, 17, 7936-7948.

(55) Lee, E. H.; Byrn, S. R. Stabilization of Metastable Flufenamic Acid by Inclusion of Mefenamic Acid: Solid Solution or Epilayer? J. Pharm. Sci. 2010, 99, 4013-4022.

(56) Lee, E. H.; Boerrigter, S. X.; Byrn, S. R. Epitaxy of a Structurally Related Compound on the (100) Faces of Flufenamic Acid Form I and III Single Crystals. Cryst. Growth Des. 2010, 10, 518-527.

(57) Engkvist, O.; Price, S. L.; Stone, A. J. Developments in computational studies of crystallization and morphology applied to urea. Phys. Chem. Chem. Phys. 2000, 2, 3017-3027.

(58) Warzecha, M.; Guo, R.; Bhardwaj, R. M.; Reutzel-Edens, S. M.; Price, S. L.; Lamprou, D. A.; Florence, A. J. Direct Observation of Templated Two-Step Nucleation Mechanism during Olanzapine Hydrate Formation. Cryst. Growth Des. 2017, 17, 6382-6393.

(59) Seaton, C. C.; Parkin, A.; Wilson, C. C.; Blagden, N. Growth of an organic co-crystal upon a component subphase. Cryst. Growth Des. 2008, 8, 363-368.

(60) Chadwick, K.; Chen, J.; Myerson, A. S.; Trout, B. L. Toward the Rational Design of Crystalline Surfaces for Heteroepitaxy: Role of Molecular Functionality. Cryst. Growth Des. 2012, 12, 1159-1166.

(61) Wijethunga, T. K.; Baftizadeh, F.; Stojakovic, J.; Myerson, A. S.; Trout, B. L. Experimental and Mechanistic Study of the Heterogeneous Nucleation and Epitaxy of Acetaminophen with Biocompatible Crystalline Substrates. Cryst. Growth Des. 2017, 17, 3783-3795.

(62) Park, Y.; Boerrigter, S. X. M.; Yeon, J.; Lee, S. H.; Kang, S. K.; Lee, E. H. New Metastable Packing Polymorph of Donepezil Grown on Stable Polymorph Substrates. Cryst. Growth Des. 2016, 16, 25522560.

(63) Lee, E. H.; Boerrigter, S. X. M.; Rumondor, A. C. F.; Chamarthy, S. P.; Byrn, S. R. Formation and solid-state characterization of a salt-induced metastable polymorph of flufenamic acid. Cryst. Growth Des. 2008, 8, 91-97.

(64) Quiñones, R.; Brown, R. T.; Searls, N.; Richards-Waugh, L. Study of polymorphism using patterned self-assembled monolayers approach on metal substrates. Appl. Surf. Sci. 2018, 427, 97-105. 